\title{
VARIACIÓN DE HABER IMPERSONAL EN EL ESPAÑOL DE LAS PALMAS DE GRAN CANARIA*
}

\author{
VARIATION OF THE IMPERSONAL HABER IN THE SPANISH \\ SPOKEN IN LAS PALMAS DE GRAN CANARIA
}

\author{
Clara Eugenia Hernández Cabrera \\ Universidad de Las Palmas de Gran Canaria \\ claraeugenia.hernandez@ulpgc.es
}

Recibido: 02/09/2016

Aceptado: 03/10/2016

\begin{abstract}
Resumen
Aportamos en este trabajo los resultados del estudio sobre la personalización de haber en el corpus PRESEEA-Las Palmas. Para ello se han analizado los 365 ejemplos registrados en los parlamentos de los 72 informantes de la muestra. Los datos reflejan que la pluralización no está tan generalizada como se indicaba en la bibliografía precedente. Para explicar la variación observada se tienen en cuenta los diez factores lingüísticos, los dos estilísticos y los tres sociales de la propuesta de codificación del proyecto PRESEEA.

PALABRAS CLAVE: Pluralización de $h a-$ ber, variación gramatical, PRESEEA, español de Canarias, Sociolingüística.
\end{abstract}

\begin{abstract}
In this article we shall present the results of the study on the personalization of the verb haber in the PRESEEA-Las Palmas corpus. We have analyzed the 365 examples registered in the discourse of the 72 speakers that make up the sample. The results show that pluralization is not as widespread_as was considered in the previous bibliography. In order to explain the variation found in the analysis, we have taken into account the factors included in the codification proposal within the PRESEEA project: 10 of them are linguistic, 2 stylistic and 3 sociological.

KEYWORDS: Pluralization of haber, morphosyntactic variation, PRESEEA, Spanish spoken in the Canary Islands, Sociolinguistics.
\end{abstract}

\footnotetext{
* Esta publicación forma parte del proyecto de investigación "Estudio complementario de los patrones sociolingüísticos del español de España-Las Palmas de Gran Canaria (ECOPASOS)" (Referencia FFI2015-68171-C55-P), financiado por el Ministerio de Economía y Competitividad y por el Fondo Europeo de Desarrollo Regional (FEDER).
}

Para citar este artículo / To cite this article: Hernández Cabrera, Clara Eugenia (2016). Variación de haber impersonal en el español de Las Palmas de Gran Canaria. ELUA, 30: 141-162. doi:10.14198/ ELUA2016.30.07

Enlace / Link: http://dx.doi.org/10.14198/ELUA2016.30.07 


\section{Introducción}

Nos proponemos en este artículo aportar los resultados del análisis de la personalización de haber en los tres niveles socioeducativos de la ciudad de Las Palmas de Gran Canaria contemplados de manera general en el proyecto PRESEEA. Para este propósito se tendrán en cuenta los diez factores de carácter lingüístico, los dos estilísticos y los tres de tipo social que se incluyen en la propuesta de codificación elaborada por varios equipos del proyecto (cf. PRESEEA 2013) ${ }^{1}$ para el estudio de la citada variable dependiente. Es importante destacar que en la reunión celebrada en Comillas en el otoño de 2010 la pluralización de haber se incorporó como uno de los objetos de estudio prioritario para los equipos internacionales del PRESEEA; constituye esto una muestra del interés por este fenómeno en el español actual, porque es necesario conocer con precisión su difusión geográfica y social. Entre otras posibilidades, cabe plantearse si, como señalan algunos investigadores, la pluralización de haber se está extendiendo geográficamente con el resultado de que hablantes de zonas que antes no presentaban tal variación hoy podrían pluralizar algunos casos de haber. Así, Navarro (1991) señala que el empleo de este verbo como unipersonal ha ido perdiendo terreno en el ámbito hispánico. De la misma forma, Díaz Campos (2003a) ve el fenómeno como un cambio en marcha en español. También la Nueva Gramática de la Lengua Española (NGLE) coincide con esta visión: "El fenómeno está hoy en expansión, con intensidad algo mayor en América que en España" (RAE/ ASALE 2009: 3063). Tal tendencia es contraria a lo que vaticinaban algunos estudiosos, para quienes la pluralización de haber estaba llamada a quedar relegada al "uso vulgar" de la lengua; recordemos en este sentido las palabras de Bello: "Es preciso corregir el vicio casi universal en Chile de convertir el complemento directo en sujeto de haber" (1981 [1847]: 467). Queda aún una tercera posibilidad: que el fenómeno se mantenga como un caso de variación estable, sin progresos ni retrocesos marcados.

Cuando se plantea el estudio de una variable gramatical siempre ha de tenerse en cuenta si la alternancia entre las formas que se pretende analizar plantea algún problema teórico para ser abordada como un caso de variación sociolingüística (vid., entre otros, Labov 1978; Sankoff 1988; Silva Corvalán 2001; López Morales 2004). Nuestros conocimientos previos nos indican que en muchas zonas del mundo hispanohablante (vid., por ejemplo, De Mello 1991) alternan las formas del singular y del plural sin que de ello se deduzca un cambio de significación, ni semántica ni pragmática. En la clasificación de Martín Butragueño (1994: 53), esta variable se sitúa entre las de tipo funcional (en cuanto supone una reinterpretación del CD como sujeto) y se caracteriza en la matriz gramatical por estar condicionada solo por factores morfológicos y sintácticos.

En nuestro corpus se puede observar la alternancia de las formas de singular y plural de haber en un mismo hablante sin que se produzca modificación alguna de la situación comunicativa; en este caso la variable se comporta de forma similar a como lo hacen las de carácter fónico, si bien es cierto que, como cabía esperar, con una frecuencia marcadamente inferior. Los siguientes enunciados son ejemplos de nuestros materiales del PRESEEA:

1 Para la elaboración de la propuesta fueron fundamentales las aportaciones de Paola Bentivoglio y José R. Gómez Molina. 
(1) y posiblemente habrán dos padres// los dos masculinos o dos padres los dos femeninos// que les den ese cariño y habrá otros que no/ por supuesto// (LP06M13) ${ }^{2}$

(2) y entonces en los laterales había como una:// como unas hornacinas empotradas en donde habían cosas relacionadas// pues vajillas o bandejas// y demás// [...] y de// después desde la zona de:/ donde estaba el piano/ había otra salita en donde habia// (e:)/ pues unos muebles empotrados donde estaban la:s// las bebidas (LP11H23).

(3) en el pasillo habian: m:uebles había sillones había de todo ¿no?/ (LP13M23).

(4) habia unos coches que se llamaban coches pulga// que eran un: (risas)/ les dabas cuerda// y salían después disparados pero dando vueltas en círculo a una velocidad tremenda/ y se llamaban coches pulga porque eran muy:/ muy pequeñitos// y luego después/ habian los juegos que nosotros nos inventábamos//( LP18H33)

(5) en cada una// en: depende/ habian: algunas que// depende de lo que: de lo que quisieras ver porque// a veces:/ (m:) habia radiografías con contraste// (LP22M33)

(6) allí tenía mi: mi padre tenía unas cabras// habían perros// gallinas// y allí vivíamos todo el mundo:/ [...] que era una habitación/ pequeña también// después la cocina// y el cuarto de baño/ después allí un: especie de un:/ un corral// que había unas cabras allí:/ (LP66H31)

Bentivoglio y Sedano (1989), en uno de los trabajos más influyentes sobre el tema, expusieron con rigor y sencillez los motivos que explican el uso plural de este verbo. De acuerdo con estas estudiosas, la pluralización de haber se ve favorecida, entre otras razones, por ser un verbo con un solo argumento, lo cual permite considerar esa FN como sujeto y no como CD, y por su valor de verbo existencial de carácter presentativo. Luque (1978), por su parte, ha explicado que el desplazamiento de habere hacia lo existencial se enmarca en una larga tradición en la que dicho verbo se ha ido empleando con un sentido y unas funciones parecidas a las de esse.

En muchas de las comunidades estudiadas son los condicionantes sociales los que explican fundamentalmente la variación observada. Así ocurre en general, según Blas Arroyo (2005: 32-33), en las zonas españolas monolingües donde se registra la pluralización, una situación que contrasta con aquellas donde se produce el contacto con el catalán, en las que el fenómeno puede interpretarse como producto de la convergencia gramatical con dicha lengua. Naturalmente, también influyen en tal variación ciertos factores lingüísticos, como los que indican Bentivoglio y Sedano (2011: 173-174), aunque resulta muy sorprendente que la incidencia de los contemplados en las cuatro investigaciones hispanoamericanas que comentan en su artículo (las de Díaz Campos 2003a; D’Aquino 2004; Castillo Trelles 2007 y Freites 2008) sea tan diferente en las comunidades analizadas; este resultado invita a un estudio detallado del fenómeno en otras zonas con el fin de tratar de encontrar posibles generalizaciones.

\section{Haber en Canarias. Estudios previos}

Se tiene la idea de que el uso de las formas personales del verbo haber es frecuente en el español de las islas. En una de las consultas formuladas a la Academia Canaria de la Lengua, un hablante isleño pregunta lo siguiente:

2 Tras cada ejemplo se registra el código de la ciudad (LP), el número de la entrevista, el sexo del informante (H o M), su edad (generaciones 1, 20-34 años, 2, 35-54 años, y 3, 55 y más años) y su nivel educativo (1, primario, 2, secundario, y 3, universitario). 
Muchas de las personas que trabajan conmigo y yo mismo, hacemos uso de expresiones como: "ese año hubieron problemas" o "habían más de 200 personas". En castellano este uso del verbo "haber" es incorrecto. ¿Es una característica del habla canaria o simplemente hablamos incorrectamente? (Consulta núm. 134).

También los investigadores del español de Canarias han considerado, de forma casi unánime, que el uso personal de haber en las islas es un fenómeno generalizado, incluso entre hablantes de los niveles sociales más altos. Son muy elocuentes las siguientes afirmaciones: "Frases como ¿Cuántas personas hubieron?, El lunes hubieron fiestas, Habian niños jugando en la calle, son naturalísimas en boca de personas universitarias" (Catalán 1964: 247); "está tan enraizado en Canarias, que hemos visto y oído concordancias de este tipo hasta en escritos y conferencias de profesores universitarios isleños" (Régulo 1968-69: 69), "común en el español general de Canarias de cualquier clase social" (Álvarez Nazario 1972: 91), "está difundidísimo entre todas clases de gentes" (Alvar 1975-78, t. III, lám. 1201) "ampliamente difundido" [en todos los niveles culturales] (Almeida y Alayón 1989: 127).

Los estudios sobre comunidades particulares coinciden con las afirmaciones anteriores, tal como puede leerse en las monografías sobre La Palma (Régulo 1968-69: 69), Los Silos, Tenerife, (Lorenzo 1976: 104), Playa de Santiago, La Gomera (C. Alvar 1975: 81), las zonas rurales de Gran Canaria (Almeida 1989: 122), Fuerteventura (Morera 1994: 144-145) y Lanzarote (Torres Stinga 1995: 143).

El uso personal de haber es incluido por Ortega (1996: 142-144) entre los rasgos gramaticales que pertenecen a la norma culta isleña aunque se apartan del ideal de lengua general. Es también relevante el testimonio de Hernández (2004: 126) sobre el frecuente uso personal de haber en los medios audiovisuales.

A pesar de la importancia de todas estas observaciones, no existen datos cuantitativos, salvo las excepciones que indicaremos, sobre la variación de haber en las islas; por ello tienen razón Ortega (1996) y González Calvo (2007) cuando echan en falta ese tipo de trabajos para confirmar empíricamente las opiniones de los dialectólogos. Los únicos datos numéricos con los que contamos son los de Pérez Martín (2007) sobre El Hierro y los análisis previos sobre materiales parciales del PRESEEA-Las Palmas (Samper y Hernández Cabrera 2012, 2016).

$\mathrm{Si}$ atendemos a la perspectiva diacrónica, contamos con testimonios de formas personales de haber en el español de las islas desde el siglo XVII. Morera (1994: 145) aporta ejemplos extraídos de unas órdenes dictadas por el obispo Bernardo de Vicuña en 1695 contra las diversiones de los naturales de Fuerteventura: "Mandamos [...] no haian danzas, bailes ni entretenimientos de noche, ni día en casa de la parida; Mandamos no haian en la noche o día en que se celebren la boda, ni canten letras [...]". También Medina (1994-95) ha recogido el empleo constante de haber personal en un texto inédito escrito en la capital de El Hierro en 1721 por un escribano de dicha isla: "Era muy uaja mucho mas quela Ysla dela Palma y que abran dies o dozedias [...]", "y que abran veinte y cuatro dias que vio [...]".

\section{Metodología}

El estudio actual supone el análisis de las 365 ocurrencias de haber acompañado de un argumento en plural que se registran en los parlamentos de 72 sujetos, 24 de cada uno de los niveles educativos diferenciados. La muestra, que se expone en el cuadro 1, se ajusta al criterio de cuotas por afijación uniforme, como es prescriptivo dentro del proyecto: 


\begin{tabular}{|c|c|c|c|c|c|c|c|c|c|c|}
\hline \multirow[b]{3}{*}{ Generación } & \multicolumn{9}{|c|}{ Nivel de estudios } & \\
\hline & \multicolumn{3}{|c|}{ Primarios } & \multicolumn{3}{|c|}{ Secundarios } & \multicolumn{3}{|c|}{ Superiores } & \\
\hline & $\mathrm{I}$ & II & III & $\mathrm{I}$ & II & III & I & II & III & $\mathrm{N}$ \\
\hline Hombres & 4 & 4 & 4 & 4 & 4 & 4 & 4 & 4 & 4 & 36 \\
\hline Mujeres & 4 & 4 & 4 & 4 & 4 & 4 & 4 & 4 & 4 & 36 \\
\hline $\mathrm{N}$ & 8 & 8 & 8 & 8 & 8 & 8 & 8 & 8 & 8 & 72 \\
\hline
\end{tabular}

Cuadro 1. Muestra de hablantes.

La delimitación de la variable dependiente se ha realizado de acuerdo con los presupuestos metodológicos del grupo de PRESEEA que trabaja este fenómeno, lo que significa que nuestros datos van a poder compararse directamente con los de las investigaciones que se realicen en otras comunidades españolas e hispanoamericanas.

Así pues, se han contabilizado todos los casos de haber acompañado de una FN plural:

(7) a pues entonces: ibas al patio grande de arriba// o sea que habia tres patios// en: en el colegio/ (LP54M11)

b decíamos “¿no tienes:/ Tropical?”// dicen/ "no no:/ Dorada nada más”// y habian piques con eso/ pero de broma/ [¿sabes?] (LP49H11)

También se incluyen en el recuento los casos en que el CD singular representa una entidad colectiva:

(8) a siempre/había un par de zapatos que mi madre decía/ (LP33H22)

b y todos los países colindantes/ han habido una cantidad de// de casos de cáncer// que a partir de ahí/ se dispararon de una manera:/ brutal/ (LP64M21)

Se analizan asimismo los ejemplos en que el verbo figura en construcciones perifrásticas:

(9) a suele haber también// unas vigas/ también de hormigón (LP11H23)

$\mathrm{b}$ en esos gastos en ese mantenimiento en esa// digamo:s// o desarrollos// que puedan haber/ de: de de cosas nuevas/ de nuevas normativas/ (LP42H32)

Lógicamente, no se han tenido en cuenta las numerosas ocurrencias de hay porque en Las Palmas no se registra la forma pluralizada hayn, que sí se documenta en otras islas arcaizantes del Archipiélago ${ }^{4}$.

Nuestro estudio actual se limita a la tercera persona. No hemos incluido en los cálculos las formas de la primera, que son infrecuentes en los grupos alto y medio. Contamos en el nivel universitario solo con un ejemplo de habíamos, usado por una hablante de la tercera generación que, además, se corrige inmediatamente:

3 Cuando aparezcan (a) y (b) en los ejemplos, la primera corresponde al uso no concordado y la segunda al pluralizado.

4 Alvar registra en el ALEICan dos casos de hayn, uno en La Gomera y otro en El Hierro; también en Fuerteventura se oye ocasionalmente esta forma (Morera 1994). El estudio más amplio sobre hayn en el Archipiélago se lo debemos a Pérez Martín (2005) en la isla de El Hierro. Las 10 ocurrencias de este plural que documenta en sus materiales son realizadas mayoritariamente por los hombres; como cabía esperar por el carácter arcaizante de la forma, son los hablantes de la tercera generación los que hacen más uso de ella y, también como era previsible, es en los dos niveles bajos donde se produce la mayor parte de los ejemplos. 
(10)

mira/ (m:) yo qué sé habiamo:s/ estábamos un: (LP23M33)

En el nivel medio hemos registrado 2 casos:

(11) nos conocíamos/ habiamos/ había muchísimos niños (1") y: y bueno y/ (LP27H12)

(12) pues había natación (1") y entonces éramos nosotros los canarios que habiamos pocos// má:s// más los de allá de la Península ¿no?/ (LP44H32)

Más frecuentes son las apariciones en el nivel bajo:

(13) sí sí/ estábamo:s todos junto:s ¿sabe?/ en la clase habiamos chicos y chicas juntos// no era: chicos en una clase y chica:s en otro/ (LP59H21)

(14) grande// habíamos la carpintería estaba dividida en tres// estaba/ la parte de la maquinaria// los carpinteros/ y los manisadores (sic)// (LP59H21)

(15) y había que estar en cada garita/ (ruido) dos horas// como éramos doce soldados/ y:/ no doce no habiamos por lo menos veinte// para hacer cada dos horas un: relevo// (LP60H21)

(16) habiamos como:// veintipico personas ¿sabe?/ veintipico niñas en clase// (LP54M11)

(17) la gente era muy conformista con lo que tenía// o sería como antes habíamos muchos más pobres (1") que lo que hoy en día hay// (LP71M31)

Es sintomático que los ejemplos que se han recogido correspondan mayoritariamente al pretérito imperfecto, uso considerado "normal”, según Álvarez Nazario (1972: 91), frente a la percepción como "vulgar" de habemos. De esta última forma no hemos recogido ningún ejemplo en los niveles alto y medio; los únicos dos casos que se registran en nuestros materiales corresponden al nivel más bajo de instrucción, en el que también se oye otro ejemplo de pluralización en una construcción perifrástica del verbo:

(18) ya allí (e:)/ habemos cuatro// y cada uno/ sabe sus funciones (LP59H21)

(19) donde habemos ciento sesenta y tres vecinos// (LP64M21)

(20) tenemos que haber de todo en este mundo// y como le dije aquel día/ por desgra- por gra:(m:)// suerte (Dios)// cuando nos morimos vamos todos al mismo sitio// (LP61M21)

Nuestro estudio contempla las 15 variables independientes que incorpora la propuesta de codificación adoptada por los grupos del proyecto (vid. PRESEEA 2013) con la finalidad de facilitar los cotejos entre las distintas investigaciones:

(a) Lingüísticas: (1) estructura verbal, (2) rasgo semántico de la FN, (3) carácter definido/ no definido de la FN, (4) forma léxica de la FN, (5) posición de la FN, (6) marcas de pluralidad de la FN, (7) tiempo y modo verbal, (8) intercalación de elementos, (9) tipo de oración y (10) modalidad del enunciado.

(b) Estilísticas: (11) especialización (campo) y (12) tipo de discurso.

(c) Sociales: (13) sexo, (14) edad y (15) nivel de estudios.

\section{Resultados generales}

Exponemos en el cuadro 2 los resultados que ha producido el análisis de todos los hablantes de nuestra muestra: 


\begin{tabular}{|c|c|c|}
\hline Forma verbal & $\mathrm{N}$ & $\%$ \\
\hline singular & 233 & 63,8 \\
\hline plural & 132 & 36,2 \\
\hline $\mathrm{N}$ & 365 & \\
\hline
\end{tabular}

Cuadro 2. Resultados del uso de haber con FN plural.

Las cifras anteriores indican que los hablantes palmenses pluralizan un 36,2\% de las ocurrencias del verbo cuando va acompañado de una FN plural. Son datos que no confirman el carácter generalizado de la personalización de haber que han señalado los estudiosos del español de Canarias. Hemos de preguntarnos por qué se da esta disparidad con las opiniones señaladas.

Puede ocurrir que las apreciaciones impresionistas, aun las de lingüistas muy reconocidos, hayan destacado las formas más llamativas y les hayan conferido una frecuencia superior a la que realmente tienen. Recordemos, por ejemplo, el contraste entre los datos de pluralización en la norma culta bogotana y las apreciaciones de Montes Giraldo (1982), quien consideraba que las formas concordadas constituían la norma general del país, tanto de los estratos cultos como de los incultos ${ }^{5}$. Unas palabras de Vaquero (1993: 77) son muy ilustrativas a este respecto:

Es curioso ver cómo la investigación rigurosa de los fenómenos que se creen "normales" arroja resultados inesperados y hasta contradice nuestras intuiciones. Es fácil interpretar como "norma regional" lo que, precisamente, se aparta de la norma, pues las desviaciones (en el mejor sentido de la palabra) cuentan con índices de percepción más altos que los hábitos. Por eso es tan importante la investigación de los hechos, antes de adoptar decisiones respecto a lo que se cree norma local.

En esta misma idea ha insistido más recientemente González Calvo (2007): "la orientación mucho más fiable de trabajos como los ahora reseñados ${ }^{6}(\ldots)$ ha de impulsar la mesura y el rigor en las apreciaciones teóricas y normativas sobre nuestro haber".

Los datos de muchas investigaciones sobre determinadas comunidades americanas, como puede observarse en el cuadro 3, reflejan porcentajes más altos que los obtenidos en nuestras grabaciones. Así, superan abiertamente los índices de pluralización canarios los que aportan diferentes estudios sobre Venezuela (Bentivoglio y Sedano 1989; Navarro 1991; Domínguez et alii 1998; Díaz Campos 2003b; D’Aquino 2004; Freites 2008), sobre la población mexicana de Mérida (Castillo Tréllez 2007), sobre San Salvador (Quintanilla Aguilar 2009) y sobre tres capitales antillanas (Claes 2014) 7 .

En la investigación de Gómez Molina (2013) sobre la comunidad urbana de Valencia (España), el índice de pluralización de toda la muestra es de 46,2\%; este porcentaje, posiblemente influido por el contacto con el catalán, también es superior al palmense.

Si comparamos nuestros resultados con los que aporta De Mello (1991) sobre once ciudades hispánicas incluidas en el "Proyecto de estudio de la norma culta ${ }^{8}$, la capital canaria se

5 El uso impersonal de haber podría explicarse, según Montes Giraldo, por "la presión consciente de la norma exógena" (1982: 383). Sin embargo, como comenta De Mello (1991), en las grabaciones secretas de la norma culta bogotana todos los ejemplos de haber responden al esquema impersonal.

6 Se refiere a trabajos cuantitativos, como los de Bentivoglio (1991) y De Mello (1991).

7 Los datos de las tres capitales son los que aporta el autor cuando no tiene en cuenta la variación hay - hayn.

8 Tomaremos los datos del cuadro 2, "Distribución diatópica de pluralización de haber personal. Número de casos unipersonales $v s$. casos pluralizados", ya que se ajusta al criterio que hemos seguido también nosotros para la cuantificación de los resultados. 
acerca más a las americanas que a las dos peninsulares representadas en dicha investigación (Madrid y Sevilla). Los grancanarios (considerados todos los niveles educativos) pluralizan menos que los hablantes universitarios de La Paz (60\%), Lima (40\%) y Santiago de Chile (39\%); presentan un porcentaje idéntico al de Caracas $(36 \%)^{9}$, y algo superior a los de Puerto Rico (31\%) y La Habana (27\%); frente a estos, el porcentaje de formas concordadas que produjeron los bogotanos cultos (16\%) es significativamente más bajo que el isleño. En escasa proporción pluralizan mexicanos $(8 \%)$ y bonaerenses $(4 \%)$. A estos índices hispanoamericanos hemos de añadir la ausencia total de usos plurales de haber en los materiales de las ciudades españolas de Madrid y Sevilla.

\begin{tabular}{|l|c|}
\hline Comunidad de habla & $\%$ \\
\hline Mérida (México) & 53 \\
\hline Caracas (Díaz Campos) & 54 \\
\hline San Juan de Puerto Rico & 54,3 \\
\hline Caracas (Bentivoglio y Sedano, corpus CESC) & 55,3 \\
\hline La Habana & 60,6 \\
\hline Santo Domingo & 61,2 \\
\hline Caracas (D’Aquino) & 63 \\
\hline Mérida (Venezuela) & $70,7^{10}$ \\
\hline Valencia (Venezuela) & 79,3 \\
\hline San Salvador & 79,6 \\
\hline Táchira (Venezuela) & 82 \\
\hline
\end{tabular}

Cuadro 3. Porcentajes de pluralización de haber en comunidades hispanoamericanas.

\section{Las variables explicativas}

\subsection{Los condicionantes lingüísticos}

Expondremos a continuación los resultados correspondientes a las variables independientes de carácter interno. Son diez, como ya hemos señalado, los factores que se toman en consideración.

\subsubsection{Estructura verbal (verbo/construcción perifrástica)}

\begin{tabular}{|c|c|c|c|c|}
\hline & Verbo & $\%$ & $\begin{array}{c}\text { Construcción } \\
\text { perifrástica }\end{array}$ & $\%$ \\
\hline singular & 217 & 63,1 & 16 & 76,6 \\
\hline plural & 127 & 36,9 & 5 & 23,8 \\
\hline $\mathrm{N}$ & 344 & & 21 & \\
\hline
\end{tabular}

Cuadro 4. Resultados del uso de haber con FN plural según tipo de verbo.

9 El porcentaje que se aporta en el estudio de Bentivoglio y Sedano (1989) es algo más alto, 39,2\%.

10 Como señalan los autores, el porcentaje de habían es del 61,4\% en una muestra algo más amplia que la utilizada inicialmente. 
Los datos del cuadro 4 arrojan un resultado que no es el esperable, en cuanto la construcción perifrástica no favorece la pluralización del verbo. Como es sabido, el hecho de que en la perífrasis sea el verbo auxiliar, y no haber, el que porte la marca de persona y número, podría favorecer la concordancia. Ahora bien, estos datos distan mucho de ser representativos porque en nuestros materiales son muy escasos los ejemplos de construcciones perifrásticas (representan solo el 5,75\% del total de formas analizadas), rasgo que restringe la fiabilidad del porcentaje obtenido ${ }^{11}$.

(21) a sigue habiendo las mismas huelgas (LP03H13)

b suelen haber montón de curas// (LP25H12)

\subsubsection{Rasgo semántico ( \pm humano) de la FN}

Esta variable independiente ha sido analizada en investigaciones previas puesto que prácticamente todos los estudiosos (vid., entre otros, Bentivoglio y Sedano 1989; De Mello 1991 y Domínguez et alii 1998) parten de la consideración de que el rasgo [+ hum] de la FN puede favorecer la pluralización del verbo, dada su estrecha vinculación con conceptos como tópico, agente y sujeto (vid. Silva Corvalán 1983: 119). Como indican Bentivoglio y Sedano (1989), si la FN se refiere a un ser humano es más fácil que sea interpretada como agente. El rasgo [+hum] es menos frecuente en nuestras encuestas que el [-hum] ya que si este figura en 282 de los 365 casos totales, el primero limita su presencia a los 83 ejemplos restantes, es decir, el 77,3\% frente al 22,7\% del total de las apariciones de haber con FN-plural, proporción en que hay plena coincidencia con otros estudios:

(22) a o se resbala ella y se mata como ya ha habido personas que se han matado con/ (LP46M32)

b me daba miedo llegar después del cine/ porque habian prostitutas en la esquina// (LP26H12)

(23) a en mi casa// tiene que haber caracoles// (LP37M22)

b y además en esa calle pues habian varias carpinterías/ (LP27H12)

\begin{tabular}{|c|c|c|c|c|}
\hline & {$[-$ hum $]$} & $\%$ & {$[+$ hum $]$} & $\%$ \\
\hline singular & 192 & 68,1 & 41 & 49,4 \\
\hline plural & 90 & 31,9 & 42 & 50,6 \\
\hline $\mathrm{N}$ & 282 & & 83 & \\
\hline
\end{tabular}

Cuadro 5. Resultados del uso de haber con FN plural según el rasgo [+hum]/[-hum] de la FN.

Los resultados del cuadro 5 ponen de manifiesto que también en Las Palmas los hablantes, en la mitad de las ocurrencias, interpretan como sujeto la FN cuya referencia es humana y, por tanto, hacen concordar con ella la forma correspondiente de haber. Goldvarb,

11 Este resultado no es raro. En Caracas, por ejemplo, la proporción de las construcciones perifrásticas es aun menor: D’Aquino (2004) solo registra 16 ejemplos entre 754 ocurrencias de haber. 
como veremos, selecciona esta variable como significativa y da al rasgo [+hum] un peso probabilístico de .594. Se unen, pues, nuestros datos a los de Bentivoglio y Sedano (1989), De Mello (1991), Domínguez et alii (1998), Díaz Campos (2003a) ${ }^{12}$, Freites (2008) y Rivas y Brown (2012), quienes encontraron que este factor era también muy significativo ${ }^{13}$.

\subsubsection{Carácter definido/no definido de la FN}

Como señala Bentivoglio (1993), dado que los sujetos son mayoritariamente definidos y los OD, indefinidos, el que haber vaya acompañado de una FN definida podría propiciar la concordancia en plural. La casi absoluta inexistencia de FN plurales con carácter definido en los materiales que trabajamos invalida cualquier conclusión sobre la incidencia de esta variable entre los hablantes de Las Palmas e impide confirmar si la FN definida favorece efectivamente la concordancia plural. Solo hemos contabilizado cuatro ejemplos en que el núcleo va acompañado de artículo determinado, que representan un $1,1 \%$ del total, dato cuantitativo que está en consonancia con lo señalado por Fernández Soriano y Táboas (1999: 1755) sobre la necesaria combinación de haber con sintagmas nominales indefinidos.

(24) a y habia las puertas abiertas y eso (LP01H13)

b por la avenida de: Mesa y López// por ahí// que antes habian las arenas:/ (LP63M21)

\begin{tabular}{|c|c|c|c|c|}
\hline & Definido & $\%$ & No definido & $\%$ \\
\hline singular & 2 & 50 & 231 & 64 \\
\hline plural & 2 & 50 & 130 & 36 \\
\hline $\mathrm{N}$ & 4 & & 361 & \\
\hline
\end{tabular}

Cuadro 6. Uso de haber con FN plural según el carácter definido/no definido de la FN.

\subsubsection{Forma léxica de la FN}

La propuesta de codificación adoptada por los equipos del proyecto distingue entre los casos en que el núcleo de la FN es un sustantivo de aquellos otros en que es un pronombre (personal o relativo); una tercera posibilidad es que esa FN sea tácita o implícita, como en los ejemplos siguientes:

(25) a no había barreras// y: estamos: ¿sabe?/ y nos miramos nosotros// y no creo que:// que debería haber/ (LP54M11)

b no no/ sí habian/ sí habian// no tantos como ahora/ (LP44H32)

La frecuencia más alta corresponde a las frases cuyo núcleo es un sustantivo; es mucho menor la aparición de las FN con núcleo pronominal y de las tácitas (solo un 9,3\% y un $9,9 \%$, respectivamente, del total).

12 Así fue en el caso de Caracas, pero no en Santiago de Chile. Tampoco resultó significativa en su comparación de dos corpus caraqueños (Díaz Campos 2003b).

13 No lo fue, sin embargo, en el estudio de D'Aquino (2004), aunque el rasgo [+ hum] favorece ligeramente la personalización del verbo (64\% vs. $62 \%)$. 


\begin{tabular}{|c|c|c|c|c|c|c|}
\hline & sustantivo & $\%$ & pronombre & $\%$ & tácito & $\%$ \\
\hline singular & 191 & 64,7 & 27 & 79,4 & 15 & 41,7 \\
\hline plural & 104 & 35,3 & 7 & 20,6 & 21 & 58,3 \\
\hline $\mathrm{N}$ & 295 & & 34 & & 36 & \\
\hline
\end{tabular}

Cuadro 7. Resultados del uso de haber con FN plural según la forma léxica de la FN.

El cuadro 7 muestra con claridad que la única variante que favorece la pluralización -y lo hace en una proporción relativamente elevada- es la FN tácita, mientras que la forma pronominal la frena especialmente.

Con respecto a los estudios de D'Aquino (2004) y de Freites (2008), que son de los pocos investigadores que analizan este condicionante, aunque con ligeras diferencias en las variantes que contemplan, coinciden nuestros datos en que las formas pronominales son las que más restringen la pluralización de haber, algo fácilmente explicable sobre todo en aquellos casos en que el pronombre es los, las, dada la diferenciación entre las formas tónicas y átonas del pronombre personal.

\subsubsection{Posición de la FN}

\begin{tabular}{|c|c|c|c|c|c|c|}
\hline & antepuesta & $\%$ & pospuesta & $\%$ & elidida & $\%$ \\
\hline singular & 30 & 75 & 199 & 62,8 & 4 & 50 \\
\hline plural & 10 & 25 & 118 & 37,2 & 4 & 50 \\
\hline $\mathrm{N}$ & 40 & & 317 & & 8 & \\
\hline
\end{tabular}

Cuadro 8. Resultados del uso de haber con FN plural según la posición de la FN.

El cuadro 8 confirma que la FN que acompaña a haber suele aparecer pospuesta, de acuerdo con el valor existencial y presentativo del verbo (cfr. Contreras 1978; Bentivoglio 1989); en nuestros materiales, esto ocurre en el $86,8 \%$ de las ocasiones. De las otras dos posibilidades, la posición antepuesta, que representa el 11\% del total, favorece, curiosamente, la forma singular, a pesar de que se podía esperar un índice superior de pluralización puesto que coincide con el orden más general en español para la combinación sujeto-verbo.

(26) a españoles había un montón (LP02H13)

b aquí accidentes (m:) no han habido// (LP67H31)

En cuanto a las FN elididas, su frecuencia es tan reducida en nuestro corpus que la proporción de aparición de cada una de las variantes carece totalmente de relevancia.

(27) a [yo creo] que va a seguir habiendo (LP08M13)

b tampoco se prevé/ se preveía/ ni se prevé ahora// que vaya a que que fuesen a haber (e:)/ en el futuro// (LP42H32) 


\subsubsection{Marca de pluralidad de la FN}

\begin{tabular}{|c|c|c|c|c|c|c|}
\hline & sin modificadores & $\%$ & con modificadores & $\%$ & colectivo & $\%$ \\
\hline singular & 112 & 64 & 111 & 62,4 & 10 & 83,3 \\
\hline plural & 63 & 36 & 67 & 37,6 & 2 & 16,7 \\
\hline $\mathrm{N}$ & 175 & & 178 & & 12 & \\
\hline
\end{tabular}

Cuadro 9. Resultados del uso de haber con FN plural según las marcas de la pluralidad de la FN

No hay diferencias, prácticamente, entre las FN que están constituidas por un sustantivo solo, sin acompañamiento de modificadores, y aquellas otras en que el núcleo nominal se acompaña de algún tipo de modificador o adyacente, con refuerzo de la idea de pluralidad. Tanto su representación en el corpus (47,9\% y 48,8\%, respectivamente, de los casos) como su porcentaje de pluralización ( $36 \%$ y $37,6 \%$ para cada uno) son similares. Sí hay contraste con los datos que arrojan los casos en que la FN es una entidad colectiva (es decir, un sustantivo cuantificativo especificado, del tipo cantidad, montón o serie) porque en este caso desciende el número de personalizaciones de haber, a pesar de que se trata de un contexto que normalmente favorece el plural; ahora bien, el escaso índice de aparición de los colectivos (solo 12 ocurrencias) impide dar significación a este resultado.

\subsubsection{Tiempo y modo verbal}

Se produce una gran coincidencia de los datos correspondientes a esta variable con muchas de las investigaciones realizadas en distintas comunidades hispánicas. Los resultados de nuestro análisis son los siguientes:

\begin{tabular}{|l|c|c|c|c|c|}
\hline & singular & $\%$ & plural & $\%$ & $\mathrm{~N}$ \\
\hline presente de indicativo & 13 & 81,3 & 3 & 18,7 & 16 \\
\hline imperfecto indicativo & 163 & 61,3 & 103 & 38,7 & 266 \\
\hline pretérito indefinido & 12 & 85,7 & 2 & 14,3 & 14 \\
\hline perfectos comp. ind. & 12 & 66,7 & 6 & 33,3 & 18 \\
\hline futuro & 5 & 62,5 & 3 & 37,5 & 8 \\
\hline condicional & 6 & 100 & 0 & - & 6 \\
\hline presente de subjuntivo & 16 & 55,2 & 13 & 44,8 & 29 \\
\hline perfectos de subjuntivo & - & & - & - & - \\
\hline imperfecto de subjuntivo & 6 & 75,0 & 2 & 25,0 & 8 \\
\hline $\mathrm{N}$ & 233 & & 132 & & 365 \\
\hline
\end{tabular}

Cuadro 10. Resultados del uso de haber con FN plural según tiempo y modo verbal. 
Los datos del cuadro 10 permiten obtener determinadas conclusiones:

(a) El tiempo que predomina llamativamente es el imperfecto de indicativo, pues representa el $72,9 \%$ del total de los tiempos analizados. El resto de las formas tiene una presencia muy limitada en el corpus; la segunda en frecuencia es el presente de subjuntivo, con 29 ocurrencias, el 7,9\% del conjunto. La superioridad numérica del imperfecto se repite en todos los corpus analizados en las distintas comunidades ${ }^{14}$. En Caracas, Bentivoglio y Sedano, tras comprobar el superior uso de esta forma (entre el 61\% y el 76\% en los dos subcorpus que manejan), decidieron "limitar el análisis de las variables lingüísticas a las formas habia/habian" (1989: 73)15.

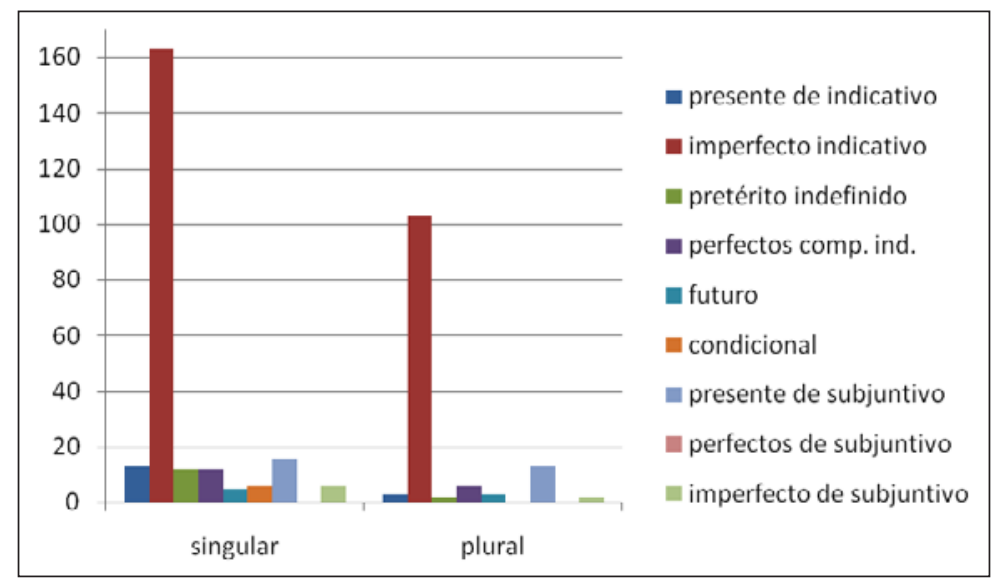

Gráfico 1. Frecuencia de los tiempos verbales en construcciones con haber y FN plural.

(b) El pretérito indefinido no favorece la pluralización ${ }^{16}$, lo mismo que ocurre en Caracas, según las investigaciones de D’Aquino (2004) y de Díaz Campos (2003b), y en San Salvador, de acuerdo con los datos de Quintanilla Aguilar (2009). Esto confirma la hipótesis de que hubieron, por su prominencia fónica, debería ser menos frecuente que las formas en que el plural se distingue solo por la nasal. Como se indica en el Manual de la NGLE: "Se ha observado estadísticamente que la concordancia es menos frecuente con el pretérito perfecto simple (hubieron) que con los demás tiempos verbales" (RAE/ASALE 2010: 779). Nuestros resultados no coinciden, sin embargo, con la situación sanjuanera, donde Rivas y Brown (2012) encuentran una proporción de hubieron que supera (58\%) a la que obtiene hubo (42\%).

(c) Particulariza a la modalidad grancanaria el peso que adquiere el presente de subjuntivo en la realización de la forma no canónica del verbo (su proporción de pluralización es la más alta de todos los tiempos, un 44,8\%). Es un dato que contrasta con los resultados que aportan Rivas y Brown (2012) para San Juan de Puerto Rico, ya que los 4 casos de su corpus se realizan, invariablemente, con la forma del singular ${ }^{17}$. Y lo mismo podemos

14 En Táchira, por ejemplo, representa el 75\% del total de ejemplos que analiza Freites (2008).

15 Es la misma decisión que después adopta Pérez Martín (2007) en su estudio de El Hierro.

16 En nuestro corpus solo aparecen dos ejemplos de hubieron, que se registran en el nivel bajo.

17 Es cierto que son pocos casos, pero llama la atención ese $100 \%$ de formas singulares, ya que es el único tiempo que alcanza esta proporción. 
decir en relación con la situación caraqueña, en la que también son mayoría las formas del singular (un $70 \%$ en un total de 10 ocurrencias), según el estudio de Bentivoglio y Sedano $(1989)^{18}$.

En las formas del presente de subjuntivo se han contabilizado, por supuesto, tanto las realizaciones estándares (haya) como las no normativas (haiga). Los cinco ejemplos de haiga en nuestro corpus, localizados todos en el nivel de instrucción bajo entre hablantes de la primera y la segunda generación, alternan la pluralización con la realización normativa:

(28) a yo creo que es que aquí no es que no haiga cantantes buenos/ (LP51H11)

b si quiere cargar esa mercancía// tiene que contratar mano portuaria// en tanto en cuanto coge/ todo los camiones que haigan fuera del muelle// y le dices que abandonen el puerto (LP57H21)

\subsubsection{Intercalación}

\begin{tabular}{|c|c|c|c|c|}
\hline & Ausencia & $\%$ & Presencia & $\%$ \\
\hline singular & 218 & 63,2 & 15 & 75 \\
\hline plural & 127 & 36,8 & 5 & 25 \\
\hline $\mathrm{N}$ & 345 & & 20 & \\
\hline
\end{tabular}

Cuadro 11. Resultados del uso de haber con FN plural según presencia/ausencia de elementos intercalados.

Como en el caso de otras variables que venimos estudiando, la desproporción numérica entre las dos posibilidades, con una escasa presencia de construcciones con elementos intercalados entre haber y la FN en plural, resta valor a los datos frecuenciales y nos impide comprobar si la intercalación de elementos entre el verbo y la FN propicia la pluralización en la comunidad grancanaria. En nuestra muestra la presencia de formas intercaladas desfavorece la pluralización, pero es un dato que se extrae solo de un 5,5\% de los ejemplos analizados.

\subsubsection{Tipo de oración}

\begin{tabular}{|c|c|c|c|c|c|c|c|c|}
\hline & independiente & $\%$ & coordinada & $\%$ & $\begin{array}{c}\text { Subordinada } \\
\text { no adjetiva }\end{array}$ & $\%$ & $\begin{array}{c}\text { Subordinada } \\
\text { adjetiva }\end{array}$ & $\%$ \\
\hline singular & 84 & 56,4 & 38 & 63,3 & 76 & 71,7 & 35 & 70 \\
\hline plural & 65 & 43,6 & 22 & 36,7 & 30 & 28,3 & 15 & 30 \\
\hline $\mathrm{N}$ & 149 & & 60 & & 106 & & 50 & \\
\hline
\end{tabular}

Cuadro 12. Resultados del uso de haber con FN plural según tipo de oración.

Son las oraciones independientes las que propician el uso plural de haber $(43,6 \%)$, mientras que las subordinadas, de cualquier tipo, presentan los promedios más bajos de personalización.

18 En otros estudios, como el de Quintanilla Aguilar (2009), la presencia del presente de subjuntivo es tan poco relevante que sus ocurrencias se suman a las de otros tiempos minoritarios bajo el rótulo "Otros", en contraste con el pretérito imperfecto y con el indefinido. 


\subsubsection{Modalidad del enunciado}

\begin{tabular}{|c|c|c|c|c|c|c|}
\hline & afirmativa & $\%$ & negativa & $\%$ & otras & $\%$ \\
\hline singular & 198 & 63,1 & 35 & 70 & 0 & - \\
\hline plural & 116 & 36,9 & 15 & 30 & 1 & 100 \\
\hline $\mathrm{N}$ & 314 & & 50 & & 1 & \\
\hline
\end{tabular}

Cuadro 13. Resultados del uso de haber con FN plural según modalidad del enunciado.

Tampoco esta variable resulta rentable para explicar la variación de haber en Las Palmas de Gran Canaria. Las enunciativas negativas (que representan solo un 13,7\% del total) no implican una mayor proporción de formas personalizadas que las que se producen en las enunciativas afirmativas (al contrario, desciende casi 7 puntos el porcentaje de pluralización), resultado que coincide con el que obtuvo D’Aquino (2004) en Caracas. El resto de las modalidades del enunciado (interrogativas, exhortativas, dubitativas, exclamativas, desiderativas...) no tiene casi representación en nuestros materiales.

\subsection{Los factores estilísticos}

\subsubsection{Especialización (campo). Tema de conversación}

No influye en la pluralización de haber el que el tema de la conversación sea técnico o no lo sea, independientemente de que el discurso técnico solo representa el 22,7 del total de apariciones del verbo en el contexto estudiado. Los porcentajes de pluralización son muy similares. No hemos registrado en nuestro corpus ningún ejemplo de haber en un enunciado de carácter metalingüístico.

\begin{tabular}{|c|c|c|c|c|}
\hline & No técnico & $\%$ & Técnico & $\%$ \\
\hline singular & 179 & 63,5 & 54 & 65,1 \\
\hline plural & 103 & 36,5 & 29 & 34,9 \\
\hline $\mathrm{N}$ & 282 & & 83 & \\
\hline
\end{tabular}

Cuadro 14. Resultados del uso de haber con FN plural según especialización.

\subsubsection{Tipo de discurso}

El cuadro 15 aporta, como puede verse, resultados de interés:

\begin{tabular}{|l|c|c|c|c|c|}
\hline & singular & $\%$ & plural & $\%$ & $\mathrm{~N}$ \\
\hline narrativo & 113 & 68,1 & 53 & 31,9 & 166 \\
\hline descriptivo & 27 & 51,9 & 25 & 48,1 & 52 \\
\hline expositivo & 54 & 65,9 & 28 & 34,1 & 82 \\
\hline argumentativo & 28 & 60,9 & 18 & 39,1 & 46 \\
\hline dialogal & 11 & 57,9 & 8 & 42,1 & 19 \\
\hline $\mathrm{N}$ & 233 & & 132 & & 365 \\
\hline
\end{tabular}

Cuadro 15. Resultados del uso de haber con FN plural según el tipo de discurso. 
Los datos de frecuencia indican que las formas plurales alcanzan el porcentaje más alto en las secuencias textuales descriptivas. Los otros tipos discursivos presentan un grado de personalización alejado de aquella, ya que el más cercano, el dialogal, se queda en un $42,1 \%$, porcentaje que está relativamente próximo al que obtiene la secuencia argumentativa $(39,1 \%)$. El discurso narrativo, el más frecuente en nuestras grabaciones, es el que mantiene más las formas canónicas $(31,9 \%)$, seguido por el expositivo $(34,1 \%)$.

Los resultados grancanarios no son sorprendentes, puesto que la secuencia descriptiva refuerza el valor existencial del verbo. También Gómez Molina (2013) encontró que en Valencia (España) este tipo discursivo alcanzaba una frecuencia muy elevada en la pluralización de haber (un significativo 73,3\%), frente a todos los demás que obtienen índices por debajo de $50 \%$, aunque luego el cálculo probabilístico no seleccionó el tipo de discurso como variable significativa. No podemos establecer más comparaciones con otras comunidades porque esta variable ha estado ausente en los estudios hispanoamericanos consultados. Confiamos en que la incorporación de este factor en la metodología del proyecto PRESEEA permita comprobar si los resultados grancanarios y valencianos pueden considerarse más o menos generales.

\subsection{Los condicionantes sociales}

\subsubsection{Nivel de estudios}

En el cuadro 16 aportamos los resultados correspondientes a esta variable independiente:

\begin{tabular}{|c|c|c|c|c|c|c|}
\hline & Nivel alto & $\%$ & Nivel medio & $\%$ & Nivel bajo & $\%$ \\
\hline singular & 124 & 79,5 & 52 & 46,4 & 57 & 58,8 \\
\hline plural & 32 & 20,5 & 60 & 53,6 & 40 & 41,2 \\
\hline $\mathrm{N}$ & 156 & & 112 & & 97 & \\
\hline
\end{tabular}

Cuadro 16. Resultados del uso de haber con FN plural según nivel educativo.

Las diferencias son muy marcadas: el nivel alto restringe fuertemente la pluralización, mientras que el medio la favorece, prefiriendo el uso concordado en algo más de la mitad de las ocurrencias $(53,6 \%)$. Lo sorprendente es que el nivel bajo presente un porcentaje de personalización perceptiblemente inferior al del medio. Se dibuja, pues, un patrón curvilíneo de estratificación en el que los grupos sociales extremos coinciden en pluralizar menos que el nivel intermedio. Se ha visto este perfil como manifestación de un cambio lingüístico en sus fases iniciales si se combina con una distribución lineal de los diferentes grupos generacionales; es necesario, pues, el estudio de la variable edad.

Muchas de las investigaciones cuantitativas realizadas en el mundo hispánico resaltan la importancia de este condicionante ${ }^{19}$ y confirman empíricamente la opinión de teóricos

19 En algunos estudios se trabaja con el nivel de instrucción mientras que en otros se contempla el nivel sociocultural, de carácter más complejo porque incorpora otros indicadores sociales. 
anteriores. Como se observa en el cuadro 17, en todas las comunidades estudiadas ${ }^{20}$, el grupo que ha tenido menos acceso a la instrucción formal es, salvo en Valencia (España), el que más favorece la realización no normativa ${ }^{21}$, y el nivel medio (con la excepción del estudio de Bentivoglio y Sedano en Caracas) suele presentar un índice de pluralización bien diferenciado del nivel superior.

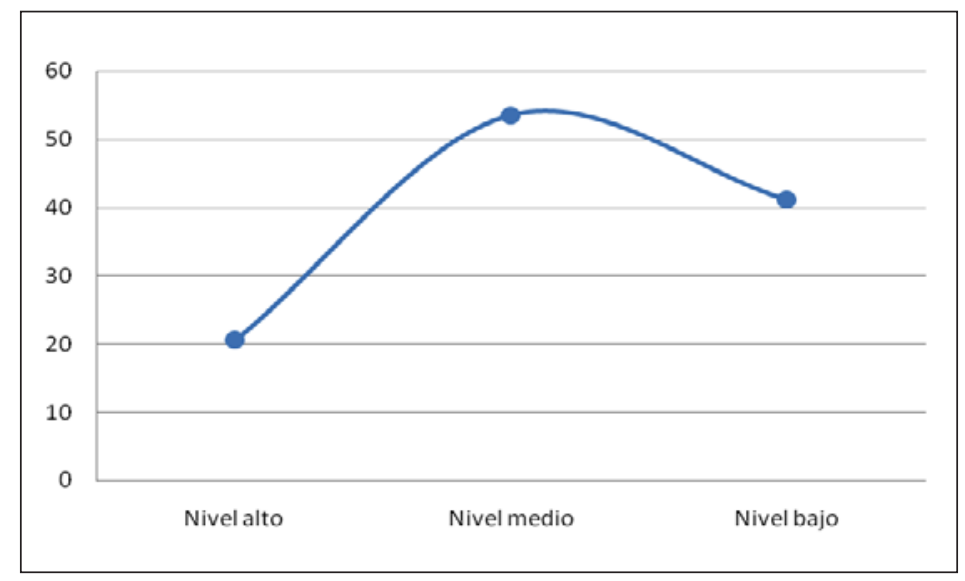

Gráfico 2. Pluralización de haber según nivel educativo.

\begin{tabular}{|l|c|c|c|}
\hline Comunidad & Nivel alto & Nivel medio & Nivel bajo \\
\hline Valencia (España) & 37 & 53,1 & 50 \\
\hline Caracas [Bentivoglio-Sedano] ${ }^{22}$ & 44,8 & 41,7 & 80 \\
\hline Caracas [D'Aquino] & 52 & $66,2^{23}$ & 70 \\
\hline Caracas (Díaz Campos) ${ }^{24}$ & 48 & 54 & 66 \\
\hline Mérida (Venezuela) & 38,8 & 62,2 & 72,5 \\
\hline Valencia (Venezuela) & 56 & 75,4 & 86,3 \\
\hline
\end{tabular}

Cuadro 17. Porcentajes de pluralización de haber en distintas comunidades de habla según nivel educativo.

20 No se tuvo en cuenta este factor en Táchira (Freites 2004). Tampoco incorporamos datos de la investigación realizada en el español yucateco por Castillo Trelles (2007) debido a que, al no ser el nivel sociocultural un factor seleccionado por Goldvarb, no se aportan los datos cuantitativos relativos a esta variable independiente.

21 También en San Salvador, donde el estudio de Quintanilla (2009) solo tuvo en cuenta dos grupos de instrucción, los hablantes con un nivel de estudios básico pluralizan más que los de nivel superior (83,5\% frente a 75,9\%).

22 Son datos que se obtienen a partir de los que incluyen las autoras en las tablas 1A y 1B, de las páginas 68 y 70 , respectivamente.

23 Hemos sacado este porcentaje sumando los correspondientes a los tres subniveles (alto, medio y bajo) que la autora diferencia dentro del nivel medio (vid. p. 15).

24 En este estudio el nivel sociocultural es uno de los dos factores seleccionados por Goldvarb como significativo. 
En este caso, como vemos, los datos grancanarios se asemejan en su distribución social a los valencianos, incluso coincide prácticamente el índice del nivel medio de ambas capitales, pero el grupo de instrucción bajo palmense se aleja más del medio que el de la capital levantina; más diferencia aún presenta el grupo universitario, muy poco "pluralizador" en Las Palmas.

\subsubsection{Sexo}

Los hombres, como indica el cuadro 18 , personalizan haber (40,5\%) más que las mujeres $(30,6 \%)$, quienes, una vez más, manifiestan el comportamiento clásico de privilegiar las formas canónicas cuando se trata de fenómenos con prestigio manifiesto.

\begin{tabular}{|c|c|c|c|c|}
\hline & $\mathrm{H}$ & $\%$ & $\mathrm{M}$ & $\%$ \\
\hline singular & 122 & 59,5 & 111 & 69,4 \\
\hline plural & 83 & 40,5 & 49 & 30,6 \\
\hline $\mathrm{N}$ & 205 & & 160 & \\
\hline
\end{tabular}

Cuadro 18. Resultados del uso de haber con FN plural según sexo.

\subsubsection{Edad}

Se observa asimismo una clara diferenciación generacional en cuanto los índices de pluralización de la tercera descienden marcadamente en las dos primeras, reflejando una regresión del fenómeno que contrasta con la afirmación de la NGLE (RAE/ASALE 2010: 3063) que habla de una expansión de la personalización de haber en el mundo hispánico. El patrón curvilíneo que se dibujaba al analizar el nivel sociocultural no se combina, pues, con un aumento de la pluralización entre las generaciones más jóvenes.

\begin{tabular}{|c|c|c|c|c|c|c|}
\hline & I & $\%$ & II & $\%$ & III & $\%$ \\
\hline singular & 82 & 70,1 & 89 & 69 & 62 & 52,1 \\
\hline plural & 35 & 29,9 & 40 & 31 & 57 & 47,9 \\
\hline $\mathrm{N}$ & 117 & & 129 & & 119 & \\
\hline
\end{tabular}

Cuadro 19. Resultados del uso de haber con FN plural según edad.

Estos resultados de índole social permiten afirmar que en Las Palmas se puede estar produciendo un cambio desde arriba, potenciado por el nivel educativo alto, las generaciones jóvenes y las mujeres, dibujando un patrón clásico en el que los grupos prestigiosos impulsan la regresión de la personalización de haber, favorecida, posiblemente, por la corrección llevada a cabo en los centros educativos.

\section{Resultados del cálculo probabilístico}

Una vez comentados los datos de actuación, recogemos los resultados que, para comprobar la significación estadística de las variables explicativas, aportó el cálculo probabilístico 
realizado con el programa Goldvarb 2001 (Robinson, Lawrence y Tagliamore 2001). En la tabla siguiente se indican las variables explicativas que han resultado significativas y los pesos probabilísticos de sus variantes.

\begin{tabular}{|l|l|c|}
\hline \multicolumn{2}{|c|}{ VARIABLES INDEPENDIENTES } & PESO PROBABILÍSTICO \\
\hline \multirow{3}{*}{ 2. Rasgo semántico de la FN } & - hum & .406 \\
\cline { 2 - 3 } & + hum & .594 \\
\hline \multirow{3}{*}{ 14. Edad } & $1^{\text {a } \text { generación }}$ & .439 \\
\cline { 2 - 3 } & $2^{\text {a } \text { generación }}$ & .442 \\
\cline { 2 - 3 } 15. Nivel de estudios & $3^{\text {a } \text { generación }}$ & .618 \\
\hline & universitario & .303 \\
\cline { 2 - 3 } & medio & .644 \\
\cline { 2 - 3 } & primario & .560 \\
\hline Input 0.418 Log likelihood $=-214.008$ Significance $=0.014$ (subida) $/ 0.083$ (bajada) \\
\hline
\end{tabular}

Cuadro 20. Variables seleccionadas por Goldvarb para explicar la pluralización de haber.

El programa solo ha seleccionado tres variables. Dos son sociales, con índices favorables de las variantes que ya destacaban en el análisis frecuencial (niveles de instrucción medio y primario, y tercera generación). De las 10 variables lingüísticas, Goldvarb ha seleccionado solo una, mientras que de las dos estilísticas ninguna ha resultado significativa.

\section{Conclusiones}

1. En nuestros materiales de PRESEEA-Las Palmas, tras el análisis de la actuación de 72 informantes de los tres niveles educativos considerados, el porcentaje de pluralización del verbo haber se sitúa en un 36,2\%. Este promedio no confirma las opiniones impresionistas que hablaban de una generalización absoluta de la personalización del verbo existencial. El índice de pluralización grancanario es inferior al que constatan todas las investigaciones realizadas en el español de Venezuela, que superan siempre el 54\% de las ocurrencias y llegan al $82 \%$ en la región de Táchira; lo mismo ocurre cuando se cotejan nuestros datos con los de Mérida (México), San Salvador y las tres capitales antillanas. El porcentaje de pluralización de Las Palmas es también menor que el que se produce en la ciudad española de Valencia.

2. Haber se pluraliza más cuando va acompañado de una FN con un referente humano $(50,6 \%)$ o cuando la $\mathrm{FN}$ es tácita $(58,3 \%)$ y cuando la forma temporal del verbo es el presente de subjuntivo $(44,8 \%)$.

3. Predominan las formas singulares, en contra de lo esperado, cuando la FN va antepuesta.

4. No hay diferencias relevantes en el uso de haber por la presencia o no de modificadores en la FN o por la modalidad del enunciado. 
5. Debido a la escasa presencia de formas perifrásticas, de FN con carácter definido y de estructuras con elementos intercalados entre el verbo y la FN, no tienen interés los resultados que arrojan las variables de carácter lingüístico tipo de verbo, carácter definido/indefinido de la FN e intercalación.

6. En lo que se refiere a los condicionantes estilísticos, el verbo tiende a su uso personal especialmente cuando se encuentra en una secuencia discursiva descriptiva.

7. En cuanto a los niveles educativos, se dibuja un patrón curvilíneo, ya que el nivel alto restringe fuertemente la pluralización (que solo alcanza un 20,5\%); el grupo de instrucción medio la favorece, prefiriendo el uso concordado en algo más de la mitad de las ocurrencias $(53,6 \%)$, un porcentaje que desciende perceptiblemente en el nivel bajo $(41,2 \%)$.

8. Son asimismo relevantes las diferencias debidas a los condicionantes sexo y edad. Los hombres propician los usos plurales más que las mujeres, con una diferencia de 9,9 puntos; en cuanto a la edad, los hablantes de la tercera generación son los que más favorecen el uso concordado, que desciende significativamente entre los hablantes más jóvenes.

9. El que el fenómeno no sea apoyado por el nivel educativo alto, por las mujeres ni por las dos primeras generaciones indica que en la comunidad grancanaria capitalina no es prestigioso y que puede estar sufriendo una regresión.

10. Las tres variables estadísticamente significativas, según el cálculo de Goldvarb, son el rasgo semántico de la FN, la edad y el nivel de estudios.

\section{Referencias bibliográficas}

Academia Canaria de la Lengua. www.academiacanarialengua.org (15-07-2016)

Almeida, Manuel (1989). El habla rural en Gran Canaria. La Laguna: Universidad de La Laguna. Almeida, Manuel y Carmen Díaz Alayón (1989). El español de Canarias. Santa Cruz de Tenerife: Ed. de los autores.

Alvar, Carlos (1975). Encuestas en Playa de Santiago (Isla de la Gomera). Las Palmas de Gran Canaria: Cabildo Insular de Gran Canaria.

Alvar, Manuel (1975-78). Atlas Lingüistico y Etnográfico de las Islas Canarias (ALEICan). Las Palmas de Gran Canaria: Cabildo Insular de Gran Canaria.

Álvarez Nazario, Manuel (1972). La herencia lingüistica de Canarias en Puerto Rico. Estudio histórico dialectal. San Juan de Puerto Rico: Instituto de Cultura Puertorriqueña.

Bello, Andrés (1981 [1847]). Gramática de la lengua castellana destinada al uso de los americanos, ed. crítica de Ramón Trujillo. Santa Cruz de Tenerife: Instituto Universitario de Lingüística Andrés Bello y Cabildo Insular de Tenerife.

Bentivoglio, Paola (1989). "Función y significado de la posposición del sujeto nominal en el español hablado". En Estudios sobre el español de América y lingüística afroamericana. Bogotá: Instituto Caro y Cuervo, pp. 40-58.

Bentivoglio, Paola (1993). "Full NPs in spoken Spanish: A discourse profile". En W. Ashby et alii (eds.). Linguistic perspectives on the romance languages. Philadelphia: John Benjamins, pp. 211-224.

Bentivoglio, Paola y Mercedes Sedano (1989). "Haber: ¿un verbo impersonal? Un estudio sobre el español de Caracas”. En Estudios sobre español de América y lingüística afroamericana. Bogotá: Instituto Caro y Cuervo, pp. 59-81. 
Bentivoglio, Paola y Mercedes Sedano (2011). "Morphosyntactic Variation in Spanish-Speaking Latin America". En M. Díaz Campos (ed.). The Handbook of Hispanic Sociolinguistics. Oxford: WileyBlackwell, pp. 168-186.

Blas Arroyo, José L. (2005). Sociolingüística del español. Desarrollos y perspectivas en el estudio de la lengua española en contexto social. Madrid: Cátedra.

Castillo Trelles, Carolina (2007). "La pluralización del verbo haber impersonal en el español yucateco". En J. Holmquist et alii (eds.). Selected Proceedings of the Third Workshop on Spanish Sociolinguistics. Somerville, MA: Cascadilla Proceedings Project, pp. 74-84.

Catalán, Diego (1964). "El español en Canarias" En Presente y futuro de la lengua española, I. Madrid: Instituto de Cultura Hispánica, pp. 239-280.

Claes, Jeroen (2014). "Sociolingüística comparada y gramática de construcciones. Un acercamiento a la pluralización de haber presentacional en las capitales antillanas", Revista Española de Lingüistica Aplicada, 27/2, pp. 338-364.

Contreras, Heles (1978). El orden de palabras en español. Madrid: Cátedra.

D’Aquino, Giovanna (2004). "Haber impersonal en el habla de Caracas. Análisis sociolingüístico", Boletín de Lingüística, 21, pp. 3-26.

De Mello, George (1991). "Pluralización del verbo haber impersonal en el español hablado culto de once ciudades", Thesaurus, 46, pp. 445-471.

Díaz Campos, Manuel (2003a). "La pluralización del verbo haber en dos áreas dialectales de Hispanoamérica”, Anuario de Lingüística Hispánica, 15-16, pp. 235-245.

Díaz Campos, Manuel (2003b). "The pluralization of haber in Venezuelan Spanish: A sociolinguistic change in real time", IU Working Papers in Linguistics 03-05: https://www.indiana.edu/ iulcwp/ pdfs/03-Diaz-Campos05.pdf.

Domínguez, Carmen L., Blanca Guzmán, Luis Moros, Maryelis Pabón y Roger Vilaín (1998). "Personalización de haber en el español de Mérida”, Lengua y Habla, 3/1, pp. 23-36.

Fernández Soriano, Olga y Susana Táboas (2000). "Construcciones impersonales no reflejas". En I. Bosque y V. Demonte (dirs.). Gramática descriptiva de la lengua española. Madrid: Espasa, pp. 1723-1778.

Freites, Francisco (2008). "Más sobre la pluralización de haber impersonal en Venezuela. El estado Táchira”, Lingua Americana, 22, pp. 36-57.

Gómez Molina, José R. (2013). "Pluralización de haber impersonal en el español de Valencia (España)", Verba, 40, pp. 253-284.

González Calvo, José M. (2007). "Tensión de normas: haber impersonal en español”. Revista de Filología de la Universidad de La Laguna, 25, pp. 249-262.

Hernández, Humberto (2004). El mensaje en los medios. A propósito del estudio lingüístico de la prensa escrita. Madrid/Frankfurt: Iberoamericana/ Vervuert.

Labov, William (1978). "Where does the sociolinguistic variable stop? A reply to B. Lavandera", Texas Working Papers in Sociolinguistics, 44. Austin: South West Educational Development Laboratory.

Lorenzo, Antonio (1976). El habla de Los Silos. Santa Cruz de Tenerife: Caja General de Ahorros de Santa Cruz de Tenerife.

López Morales, Humberto (2004). Sociolingüística, $3^{\mathrm{a}}$ ed. aumentada. Madrid: Gredos.

Luque Moreno, Jesús (1978). "En torno al sintagma 'haber impersonal + sustantivo' y sus orígenes latinos", Revista Española de Lingüística, 8/1, pp. 125-147.

Martín Butragueño, Pedro (1994). "Hacia una tipología de la variación gramatical en sociolingüística del español”, Nueva Revista de Filología Hispánica, 42/1, pp. 29-75.

Medina, Javier (1994-95). "Dialectología y diacronía en el español de Canarias: perspectivas futuras", Revista de Filología Románica, 11-12, pp. 217-236.

Montes Giraldo, José J. (1982). "Sobre el sintagma haber + sustantivo", Thesaurus, 37, pp. 383-385.

Morera, Marcial (1994). El español tradicional de Fuerteventura. La Laguna: Centro de la Cultura Popular Canaria, Cabildo Insular de Fuerteventura, Gobierno de Canarias y Ayuntamiento de Pájara. 
Navarro, Manuel (1991). "Valoración social de algunas formas verbales en el habla de Valencia (Venezuela)", Thesaurus, 46, pp. 304-314.

Ortega, Gonzalo (1996). La enseñanza de la lengua española en Canarias. Algunas perspectivas. La Laguna: Instituto de Estudios Canarios.

Pérez Martín, Ana M.a (2005). "Pluralización del verbo haber en el habla de la isla de El Hierro: datos parciales", Interlingüistica, 15, pp. 1125-1130.

Pérez Martín, Ana M. (2007). "Pluralización de había en el habla de El Hierro: datos cuantitativos", Revista de Filología de la Universidad de La Laguna, 25, pp. 505-513.

Preseea (2013). “Análisis de usos del verbo haber. Propuesta de codificación”, 25-01-2013, http:// www.linguas.net/metodología.

Quintanilla Aguilar, José R. (2009). La (des)pluralización del verbo haber existencial en el español salvadoreño: ¿un cambio en progreso?, tesis doctoral. University of Florida. < digitalcommons. butler.edu/facsch_papers/411>.

Real Academia Española y Asociación de Academias de la Lengua Española (2009). Nueva gramática de la lengua española, 2 vols. Madrid: Espasa Libros.

Real Academia Española y Asociación de Academias de la Lengua Española (2010). Nueva gramática de la lengua española. Manual. Madrid: Espasa Libros.

Régulo, Juan (1968-69). "Notas acerca de la isla de La Palma", Revista de Historia Canaria, 32, pp. 12-174.

Rivas, Javier y Esther Brown (2012). "Concordancia variable con haber en español puertorriqueño", Boletín de Lingüistica, 24, pp. 102-118.

Robinson, John; Helen Lawrence y Sali Tagliamonte (2001). GOLDVARB 2001. A multivariate analysis application for Windows, University of Cork, Department of Languages and Linguistics.

Samper Padilla, José A. y Clara E. Hernández Cabrera (2012). "En torno a los usos personales de haber en el español de Las Palmas de Gran Canaria" En T. Jiménez Juliá et alii (eds.). Cum corde et in nova grammatica. Estudios ofrecidos a Guillermo Rojo. Santiago de Compostela: Universidad de Santiago de Compostela, pp. 743-754.

Samper Padilla, José A. y Clara E. Hernández Cabrera (2016). "La pluralización de haber en dos niveles educativos de Las Palmas de Gran Canaria”. En A.M. Bañón et alii (eds.). Oralidad y análisis del discurso. Homenaje a Luis Cortés Rodríguez. Almería: Universidad de Almería, pp. 577-593.

Sankoff, David (1988). "Sociolinguistics and syntactic variation". En F. Newmeyer (ed.). Linguistics: The Cambridge Survey, vol. IV. Language: The socio-cultural context. Cambridge: Cambridge University Press, pp. 140-161.

Silva Corvalán, Carmen (1983). "On the interaction of word order and intonation: Some OV constructions in Spanish”. En F. Klein Andreu (ed.). Discourse perspective on syntax. New York: Academic Press, pp. 117-40.

Silva Corvalán, Carmen (2001). Sociolingüistica y pragmatica del español. Washington, D.C.: Georgetown University Press.

Torres Stinga, Manuel (1995). El español hablado en Lanzarote. Arrecife: Cabildo Insular de Lanzarote.

Vaquero, María (1993). "Norma lingüística y enseñanza de la lengua". En Actas del I Congreso Internacional sobre la enseñanza del español. Madrid: CEMIP, pp. 67-93. 